\title{
Correlation between Alzheimer's Disease and Dementia with Lewy Bodies Scores Using VSRAD Advance
}

\author{
Yukinori Okada1,2,3* , Norikazu Ohno ${ }^{4}$, Koji Tanaka ${ }^{5}$ \\ ${ }^{1}$ Department of Radiology, Iga City General Hospital, Iga, Japan \\ ${ }^{2}$ Department of Radiology, St. Marianna University School of Medicine, Kawasaki, Japan \\ ${ }^{3}$ Expert Imaging and Interventional Support, Shinyokohama, Japan \\ ${ }^{4}$ Ohno Medical Clinic, Iga, Japan \\ ${ }^{5}$ Department of General Medicine/Surgery, Iga City General Hospital, Iga, Japan \\ Email: *igaueno512@yahoo.co.jp
}

How to cite this paper: Okada, Y., Ohno, N. and Tanaka, K. (2021) Correlation between Alzheimer's Disease and Dementia with Lewy Bodies Scores Using VSRAD Advance. Advances in Alzheimer's Disease, 10, 33-45.

https://doi.org/10.4236/aad.2021.103003

Received: June 3, 2021

Accepted: July 12, 2021

Published: July 15, 2021

Copyright (c) 2021 by author(s) and Scientific Research Publishing Inc. This work is licensed under the Creative Commons Attribution International License (CC BY 4.0).

http://creativecommons.org/licenses/by/4.0/

\begin{abstract}
The objective of the study was to explore the relationship between the indicators of Alzheimer's disease and dementia with Lewy bodies using the voxel-based specific regional analysis system for Alzheimer's Disease (VSRAD) advance. Among 36 patients with suspected dementia, patients with Alzheimer's disease and dementia with Lewy bodies were identified using VSRAD advance from March 1 to October 30, 2019. All patients underwent brain Magnetic Resonance Imaging (MRI). We diagnosed Alzheimer's disease using Volume of Interest (VOI) in the Medial Temporal Lobe (MTL) atrophy ratio $>2$ and dementia with Lewy bodies using both VOI in the MTL atrophy ratio $\leq 2$ and gray/white matter atrophy ratio $\geq 0.2$. The correlation between the indicators of Alzheimer's disease and dementia with Lewy bodies was calculated. The number of patients classified as having Alzheimer's disease and dementia with Lewy bodies was 25 and 11, respectively. In the Alzheimer's disease group, the correlation coefficient between the extent of gray matter atrophy and the severity of atrophy in the dorsal brainstem gray matter was $r=-0.40(p=0.045)$. In dementia with Lewy bodies group, the correlation coefficient between the extent of gray matter atrophy and the severity of atrophy in the dorsal brainstem white matter was $r=-0.78(\mathrm{p}<0.01)$. Using VSRAD advance, gray matter atrophy and dorsal brainstem grey/white matter atrophy were found to be negatively correlated in Alzheimer's disease and dementia with Lewy bodies.
\end{abstract}

\section{Keywords}

Alzheimer's Disease, Dementia with Lewy Bodies, Dorsal Brainstem, Gray 


\section{Introduction}

Dementia is an urgent problem faced by the elderly. In 2002, an epidemiological study conducted in Tajiri, Miyagi Prefecture on persons $\geq 65$ years old reported an incidence of dementia of $8.5 \%$ [1]. However, the current number of patients with dementia has likely increased. Previously, the reported overall prevalence rates of various diseases among people aged $\geq 65$ years were as follows: $3.8 \%$ for dementia, 2.1\% for Alzheimer's Disease (AD), 1.0\% for vascular dementia, and $0.7 \%$ for other types of dementia [2]. However, a recent epidemiological study in Hisayama, Fukuoka Prefecture, reported the following prevalence rates: $32.3 \%$ for $\mathrm{AD}, 14.6 \%$ for vascular dementia, $9.5 \%$ for Dementia with Lewy Bodies (DLB), and $3.1 \%$ for mixed dementia [3]. Additionally, a study reported the involvement of denatured proteins, a type of protein and $\alpha$-synuclein believed to be related to the occurrence of $\mathrm{AD}$, in the onset of $\mathrm{AD}$ and $\mathrm{DLB}$ [4]. $\alpha$-synuclein is thought to be related to the occurrence of DLB [5] [6]. As the prognosis of DLB is worse than that of $\mathrm{AD}$, there exists a need to differentiate between the two diseases [6]. In imaging diagnosis, nuclear medicine is used to differentiate between $\mathrm{AD}$ and $\mathrm{DLB}$, since the reported cardiac uptake of 123I-Metal-Iodobenzylganidine (MIBG) is decreased in DLB [7]. In Parkinson's disease and DLB, tyrosine hydroxylase immunoreactive nerve fibers are defective [7]. A loss of the dopamine transporter causes a decreased uptake of 123I-ioflupane in the basal ganglia [8].

Magnetic Resonance Imaging (MRI) is used to diagnose dementia. Particularly, the voxel-based specific regional analysis system for Alzheimer's Disease (VSRAD) software developed by Eisai Co., Ltd. in Tokyo uses voxel-based morphometry to compare the data of normal individuals with that of the test subjects, calculate the $\mathrm{Z}$ score using standard deviation, and quantify the severity of cerebral atrophy, if any. The accuracy of VSRAD in distinguishing between normal individuals and AD patients has been reported to be $87 \%$ [9]. Furthermore, using the characteristic atrophy in the amygdala and hippocampus, VSRAD can distinguish between normal individuals and AD patients at a probability of $\geq 90 \%$ [10]. Recent reports also state that it can distinguish between AD and DLB [11]. In Japan, VSRAD received medical device approval as VSRAD advance (in Katakana) in 2020. Although VSRAD is used in practice, the relationship between the indicators of $\mathrm{AD}$ and $\mathrm{DLB}$, and whether they should be suspected by VSRAD, is still unclear. Therefore, this study aimed to assess the relationship between the indicators of $\mathrm{AD}$ and DLB using VSRAD advance.

\section{Methods}

\subsection{Study Design}

We used a single-center retrospective case-controlled study design. We obtained 
all cases of non-contrast enhanced brain MRI (simple brain MRI) for suspected dementia at the hospital where the authors worked from March 1 to October 30, 2019. We further selected suspected cases of AD and DLB using the scores obtained through VSRAD advance.

The Philips Ingenia 1.5T (Tokyo, Japan) was used for brain MRIs. The following images were examined for each case: diffusion $(b=0, b=1000)$, T1 - and T2-weighted, fluid-attenuated inversion recovery, susceptibility-weighted, Arterial-Spin Labeling (ASL), and Magnetic Resonance Angiography (MRA). The brain MRI images were visually evaluated by the radiologist (Y.O.). The radiologist (Y.O.) is a board-certified radiologist and radiation oncologist (Japan Radiological Society, Japanese Society for Radiation Oncology), board-certified nuclear medicine specialist (Japan Society of Nuclear Medicine). The radiologist (Y.O.) has a specialty of radiation oncology imaging and therapy, nuclear medicine, and has experience of emergency imaging (trained from 2009-2011 in 2 hospitals, and started to work from 2018 to now at 1 hospital).

For the ASL image, we inspected the following: 1) decreased cerebral blood flow at the posterior cingulate cortex and precuneus [12]; 2) normal cerebral blood flow at the primary sensorimotor cortex (characteristic in AD under single-photon emission computed tomography [SPECT]) [13]; 3) decreased cerebral blood flow at the occipital lobe $[14]$; 4) decreased cerebral blood flow at the occipital lobe and increased blood flow at the striatum and/or thalamus [15]; and 5) decreased and preserved cerebral blood flow at the occipital lobe and preserved cerebral blood flow at the posterior cingulate gyri relative to the precunei (cingulate island sign, characteristic in DLB under SPECT) [16].

In each patient, the coronal brain MRI T1-weighted image was analyzed using VSRAD. Patients with a $\mathrm{Z}$ score exceeding a standard deviation of two were extracted and deemed outliers. For findings suggestive of $\mathrm{AD}$, the Medial Temporal Lobe (MTL) - which includes the entorhinal cortex, amygdala, and hippocampus-was set as the area of interest. We measured the following: 1) Volume of Interest (VOI) atrophy ratio (mean $\mathrm{Z}$ score $>$ threshold within the target area of interest); 2) extent of gray matter atrophy (percentage of the total gray matter area with a $Z$ score $>2$ ); 3 ) extent of VOI atrophy (percentage of area in the target area of interest with $\mathrm{Z}$ score $>2$ ); and 4) ratio of atrophy (extent of atrophy in the target area of interest and extent of gray matter atrophy if the entire brain was 1). For findings suggestive of DLB, the dorsal part of the brainstem was set as the area of interest, and the following parameters were measured: 1) ratio of VOI atrophy in the gray matter (severity of VOI atrophy in the dorsal brainstem gray matter/in the MTL gray matter); 2) ratio of VOI atrophy in the white matter (severity of VOI atrophy in the dorsal brainstem white matter/in the MTL gray matter); 3) severity of VOI atrophy in the MTL gray matter; 4) severity of VOI atrophy in the dorsal brainstem gray matter; and 5) severity of VOI atrophy in the dorsal brainstem white matter. Per a report on VSRAD diagnosis, patients with a VOI atrophy ratio $\geq 2$ were diagnosed as having $A D$, and cases with a 
VOI atrophy ratio $\leq 2$, GM VOI atrophy ratio $\geq 0.2$, and white matter VOI atrophy ratio $\geq 0.2$ were diagnosed as having DLB.

\subsection{Statistical Analysis}

The Mann-Whitney U test and Fisher's exact test were used to compare the results of the patients with suspected AD and DLB using VSRAD advance scores. The correlation coefficients of the VOI atrophy ratio, the extent of gray matter, atrophy, extent and VOI atrophy, and atrophy ratio suggested $\mathrm{AD}$. The correlation coefficients of the severity of VOI atrophy in the dorsal brainstem gray matter, the dorsal brainstem white matter, and the MTL gray matter suggested DLB. All coefficients were calculated using Spearman's rank correlation coefficient. Statistical significance was defined as $\mathrm{p}<0.05$.

\subsection{Ethical Considerations}

This study was approved by the ethics review board of the hospital where the authors work (approval number 565). All of the patients gave informed consent before they participated in the study, and they could opt-out at any time through the hospital website.

\section{Results}

\subsection{Patients}

A total of 42 patients were selected to participate in this study. Among them, 36 were included and 6 were excluded -5 due to previous cerebrovascular events clearly shown on the MRI, and 1 due to a brain tumor. Previous cerebrovascular events and brain tumor may affect the cognitive function and VSRAD score. Finally, 36 patients were selected. Using the VSRAD advance scores, 25 patients were classified as having $\mathrm{AD}$ (male: 6 , female: 19 ; mean age: $81.6 \pm 5.5$ years) and 11 as having DLB (male: 5, female: 6 ; mean age: $81.3 \pm 6.2$ years). A detailed presentation of the participants' characteristics is shown in Table 1.

\subsection{Brain MRI Findings}

Diffusion-weighted images showed no intracranial acute lesions in any of the 36 patients. Furthermore, no coarse lesions or relatively clear cerebrovascular accidents were observed.

\subsubsection{Patients Classified as Having AD}

Hippocampal atrophy was observed in the T1-weighted sagittal images of all 25 patients with AD. ASL showed decreased blood flow in 16 patients, but unchanged blood flow in 8 patients. One patient could not be evaluated. The following phenomena were observed: decreased cerebral blood flow at the posterior cingulate cortex and precuneus in 16 patients; normal cerebral blood flow at the primary sensorimotor cortex in 6 patients; decreased cerebral blood flow at the occipital lobe in 16 patients; decreased cerebral blood flow at the occipital lobe and increased blood flow at the striatum and/or thalamus in 11 patients; and 
Table 1. Summary of patient characteristics $(n=36)$.

\begin{tabular}{|c|c|c|c|}
\hline & $\begin{array}{l}\text { Alzheimer's } \\
\text { disease group } \\
\quad(n=25)\end{array}$ & $\begin{array}{l}\text { Dementia with } \\
\text { Lewy bodies group } \\
(\mathrm{n}=11)\end{array}$ & p-value \\
\hline Age (years, mean $\pm \mathrm{SD}$ ) & $81.6 \pm 5.5$ & $81.3 \pm 6.2$ & 0.78 \\
\hline \multicolumn{4}{|l|}{ Sex } \\
\hline Male & 6 & 5 & 0.25 \\
\hline Female & 19 & 6 & \\
\hline Hippocampal atrophy & 25 & 0 & $<0.01$ \\
\hline Decreased blood flow in arterial-spin labeling & 16 & 10 & 0.22 \\
\hline Microbleeding in susceptibility-weighted imaging & 7 & 3 & 1.00 \\
\hline Magnetic resonance angiography findings & 25 & 10 & 0.31 \\
\hline $\begin{array}{l}\text { Decreased cerebral blood flow at } \\
\text { posterior cingulate cortex and precuneus }\end{array}$ & 16 & 8 & 1.00 \\
\hline $\begin{array}{l}\text { Normal cerebral blood flow at the primary } \\
\text { sensorimotor cortex }\end{array}$ & 6 & 1 & 0.39 \\
\hline Decreased cerebral blood flow at the occipital lobe & 16 & 8 & 1.00 \\
\hline $\begin{array}{l}\text { Decreased cerebral blood flow at the occipital lobe } \\
\text { and increased blood flow at the striatum and/or } \\
\text { thalamus }\end{array}$ & 11 & 7 & 0.47 \\
\hline Cingulate island signs & $\begin{array}{l}\text { Present in } 8 \\
\text { cases }\end{array}$ & Present in 5 cases & 0.71 \\
\hline
\end{tabular}

cingulate island signs in 8 patients. Susceptibility-Weighted Imaging (SWI) showed microbleeding in only 7 patients, with 17 having none. One patient could not be evaluated. Overall, the MRA findings were normal in all patients (Table 1).

\subsubsection{Patients Classified as Having DLB}

No hippocampal atrophy was observed in the T1-weighted sagittal images of all 11 patients with DLB. ASL showed decreased blood flow in 10 patients, with unchanged blood flow in 1 patient. The following phenomena were observed: decreased cerebral blood flow at the posterior cingulate cortex and precuneus in 8 patients; normal cerebral blood flow at the primary sensorimotor cortex in 1 patient; decreased cerebral blood flow at the occipital lobe in 8 patients; decreased cerebral blood flow at the occipital lobe and increased blood flow at the striatum and/or thalamus in 7 patients; and cingulate island signs in 5 patients. SWI showed microbleeding in only 3 of the 11 patients, and MRA showed mild stenosis of cerebrovascular disease in 1 patient (Table 1).

\subsection{VSRAD Advance Findings}

All 36 patients were analyzed using VSRAD advance.

\subsubsection{Patients Classified as Having AD}

Regarding the indicators suggesting $\mathrm{AD}$, the mean severity of VOI atrophy was $2.9 \pm 0.7$; the mean extent of gray matter atrophy was $6.8 \pm 2.9$; the mean extent 
of VOI atrophy was $66.6 \pm 17.1$; the mean atrophy ratio was $11.2 \pm 4.4$.

Regarding the indicators suggesting DLB, the mean ratio of VOI atrophy in gray and white matter was $0.24 \pm 0.1$ and $0.1 \pm 0.1$ respectively; the severity of VOI atrophy in the MTL gray matter was $2.9 \pm 0.7$; the severity of VOI atrophy in the dorsal brainstem gray and white matter was $0.7 \pm 0.2$ and $0.3 \pm 0.3$ respectively.

In 2 of the 25 patients classified as having $\mathrm{AD}$, the ratio of $\mathrm{VOI}$ atrophy in gray and white matter was both $\geq 0.2$.

\subsubsection{Patients Classified as Having DLB}

Regarding the indicators suggesting $\mathrm{AD}$, the mean severity of VOI atrophy was $1.2 \pm 0.4$; the mean extent of gray matter atrophy was $6.1 \pm 3.5$; the mean extent of VOI atrophy was $14.2 \pm 11.7$; the mean atrophy ratio was $2.6 \pm 1.9$.

Regarding the indicators suggesting DLB, the mean ratio of VOI atrophy in gray matter was $0.9 \pm 0.7$; the mean ratio of VOI atrophy in white matter was 0.6 \pm 0.6 ; the severity of VOI atrophy in the MTL gray matter was $1.2 \pm 0.4$; the severity of VOI atrophy in the dorsal brainstem gray matter was $0.8 \pm 0.2$; the severity of VOI atrophy in the dorsal brainstem white matter was $0.5 \pm 0.2$ (Table 2).

\subsection{Comparison of Patients Classified as Having AD and DLB}

The extent of gray matter atrophy was $5.7 \%$ in patients classified as having AD, and $5.5 \%$ in patients classified as having DLB, with a non-significant difference between the two results. The severity of VOI atrophy in the dorsal brainstem gray matter was 0.6 in patients classified as having $\mathrm{AD}$ and 0.8 in those classified as having DLB; no significant difference was noted. However, the severity and extent of VOI atrophy, VOI ratio, severity of VOI atrophy in the dorsal brainstem white and gray matter, and ratio of VOI atrophy in gray and white matter were significantly different (Table 2).

Table 2. Comparison of patients with Alzheimer's disease and dementia with Lewy.

\begin{tabular}{cccc}
\hline Phenomena & $\begin{array}{c}\text { Alzheimer's } \\
\text { disease group } \\
(\mathbf{n}=25)\end{array}$ & $\begin{array}{c}\text { Dementia with } \\
\text { Lewy bodies } \\
\text { group (n = 11) }\end{array}$ & p-value \\
Severity of VOI atrophy & 2.9 & 1.3 & $<0.01^{*}$ \\
Extent of gray matter atrophy & 5.7 & 5.5 & 0.42 \\
Extent of VOI atrophy & 64.4 & 14.0 & $<0.01^{*}$ \\
VOI ratio & 10.2 & 2.8 & $<0.01^{*}$ \\
Severity of VOI atrophy in the dorsal brainstem gray matter & 0.6 & 0.8 & 0.07 \\
Severity of VOI atrophy in the dorsal brainstem white matter & 0.2 & 0.5 & $<0.01^{*}$ \\
Severity of atrophy in the medial temporal lobe gray matter & 2.9 & 1.3 & $<0.01^{*}$ \\
Ratio of VOI atrophy in gray matter & 0.2 & 0.6 & $<0.01^{*}$ \\
Ratio of VOI atrophy in white matter bodies & 0.1 & 0.4 & $<0.01^{*}$
\end{tabular}

${ }^{*}$ Indicates statistical significance. VOI-volume of interest. 


\subsection{Correlation Coefficient}

We calculated the correlation coefficient between the indicators of AD and DLB.

\subsubsection{Patients Classified as Having AD}

A correlation coefficient of $r=1.00(p<0.01)$ was noted between the severity of VOI atrophy and atrophy in the MTL gray matter and of $r=0.84(p<0.01)$ between the extent of VOI atrophy and the extent of atrophy in the MTL gray matter. As seen in Table 3, a correlation coefficient of $r=-0.40(p=0.045)$ was noted between the extent of gray matter atrophy and the severity of VOI atrophy in the dorsal brainstem gray matter (Figure 1$), r=0.5(p=0.01)$ between the

Table 3. Correlation coefficients of patients with Alzheimer's disease.

\begin{tabular}{cccc}
\hline & $\begin{array}{c}\text { Severity of atrophy in } \\
\text { the medial temporal } \\
\text { lobe gray matter }\end{array}$ & $\begin{array}{c}\text { Severity of VOI } \\
\text { atrophy in the dorsal } \\
\text { brainstem gray matter }\end{array}$ & $\begin{array}{c}\text { Severity of VOI } \\
\text { atrophy in the dorsal } \\
\text { brainstem white matter }\end{array}$ \\
Severity of VOI atrophy & $\mathrm{r}=1.00$ & $\mathrm{r}=0.30$ & $\mathrm{r}=-0.01$ \\
Extent of gray matter & $\mathrm{p}<0.01^{*}$ & $\mathrm{p}=0.15$ & $\mathrm{p}=0.94$ \\
atrophy & $\mathrm{r}=0.04$ & $\mathrm{r}=-0.40$ & $\mathrm{r}=0.01$ \\
Extent of VOI atrophy & $\mathrm{p}=0.84$ & $\mathrm{p}=0.045^{*}$ & $\mathrm{p}=0.99$ \\
& $\mathrm{r}=0.84$ & $\mathrm{r}=0.25$ & $\mathrm{r}=-0.08$ \\
VOI ratio & $\mathrm{p}<0.01^{*}$ & $\mathrm{p}=0.25$ & $\mathrm{p}=0.71$ \\
& $\mathrm{r}=0.50$ & $\mathrm{r}=0.50$ & $\mathrm{r}=-0.13$ \\
& $\mathrm{p}=0.01$ & $\mathrm{p}=0.01^{*}$ & $\mathrm{p}=0.53$ \\
\hline
\end{tabular}

${ }^{*}$ Indicates statistical significance. VOI, volume of interest.

\section{Severity of VOI atrophy in the dorsal brainstem gray matter}

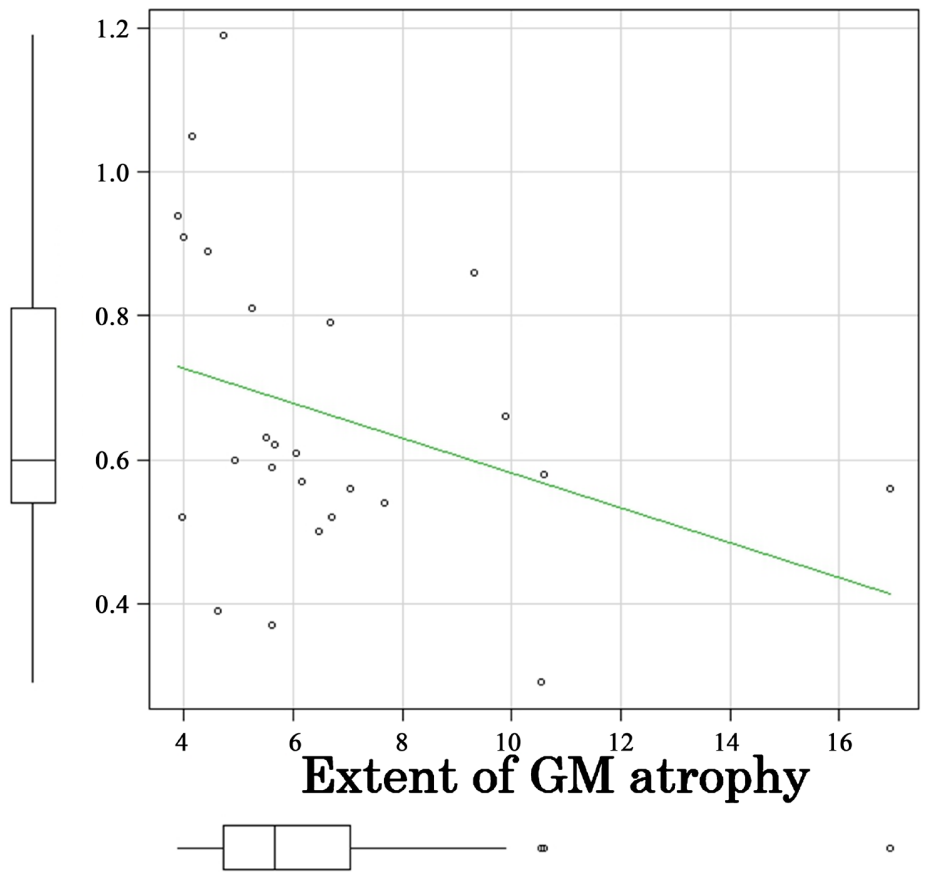

Figure 1. A correlation between the extent of gray matter atrophy and the severity of VOI atrophy in the dorsal brainstem gray matter. 
atrophy ratio and the severity of VOI atrophy in the MTL gray matter, and $\mathrm{r}=$ $0.50(p=0.01)$ between the atrophy ratio and the severity of VOI atrophy in the dorsal brainstem gray matter.

\subsubsection{Patients Classified as Having DLB}

A correlation coefficient of $r=1.00(p<0.01)$ was noted between the severity of VOI atrophy and atrophy in the MTL gray matter, $\mathrm{r}=0.97(\mathrm{p}<0.01)$ between the extent of VOI atrophy and atrophy in the MTL gray matter, and $\mathrm{r}=0.78$ ( $\mathrm{p}<$ 0.01 ) between the extent of gray matter atrophy and the severity of VOI atrophy in the dorsal brainstem white matter (Figure 2). No significant correlations were noted for the other factors (Table 4).

\section{Extent of GM atrophy}

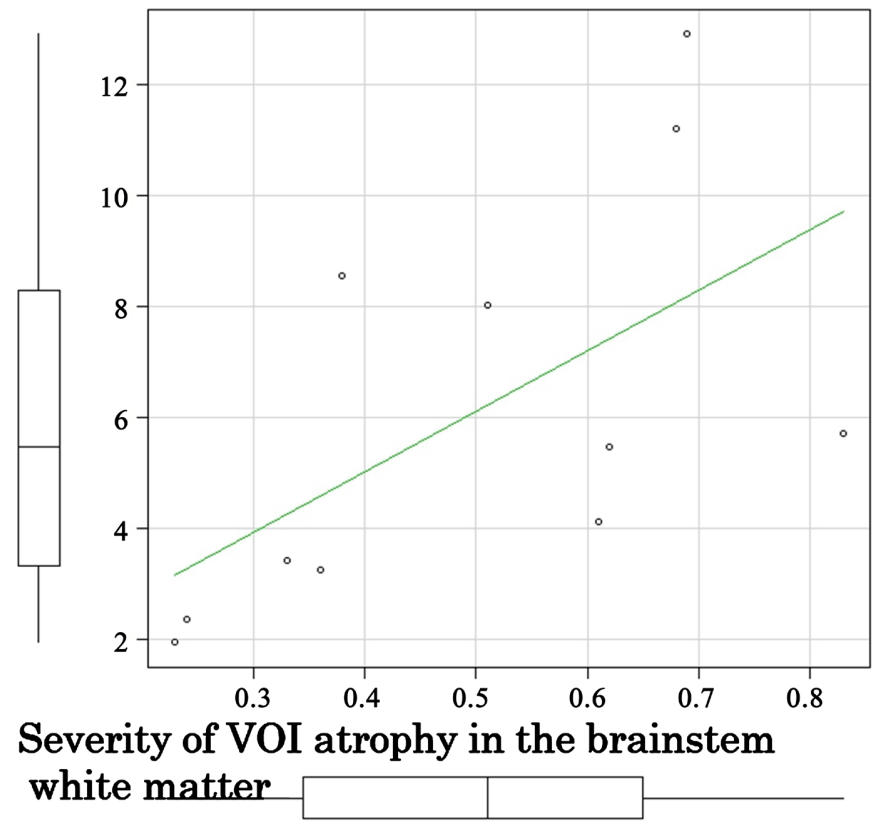

Figure 2. A correlation between the extent of gray matter atrophy and the severity of VOI atrophy in the dorsal brainstem white matter.

Table 4. Correlation coefficients of patients with dementia with Lewy bodies.

\begin{tabular}{cccc}
\hline & $\begin{array}{c}\text { Severity of atrophy in } \\
\text { the medial temporal lobe } \\
\text { gray matter }\end{array}$ & $\begin{array}{c}\text { Severity of VOI atrophy } \\
\text { in the dorsal brainstem } \\
\text { gray matter }\end{array}$ & $\begin{array}{c}\text { Severity of VOI atrophy } \\
\text { in dorsal brainstem } \\
\text { white matter }\end{array}$ \\
\hline Severity of & $\mathrm{r}=1.00$ & $\mathrm{r}=-0.39$ & $\mathrm{r}=0.07$ \\
VOI atrophy & $\mathrm{p}<0.01^{*}$ & $\mathrm{p}=0.24$ & $\mathrm{p}=0.84$ \\
Extent of GM & $\mathrm{r}=0.56$ & $\mathrm{r}=-0.24$ & $\mathrm{r}=0.78$ \\
atrophy & $\mathrm{p}=0.08$ & $\mathrm{p}=0.49$ & $\mathrm{p}<0.01^{*}$ \\
Extent of & $\mathrm{r}=0.97$ & $\mathrm{r}=-0.48$ & $\mathrm{r}=0.04$ \\
VOI atrophy & $\mathrm{p}<0.01^{*}$ & $\mathrm{p}=0.14$ & $\mathrm{p}=0.91$ \\
& $\mathrm{r}=0.54$ & $\mathrm{r}=-0.25$ & $\mathrm{r}=-0.55$ \\
VOI ratio & $\mathrm{p}=0.09$ & $\mathrm{p}=0.47$ & $\mathrm{p}=0.08$
\end{tabular}

*Indicates statistical significance. VOI-volume of interest. 


\section{Discussion}

Dementia is an important health issue that continues to affect the elderly. However, with a worse prognosis for DLB than for $\mathrm{AD}$, there is a need to differentiate between the two. Therefore, this study examined the scores of patients classified as having $A D$ and DLB using VSRAD advance to differentiate between their indicators. DLB is characterized by more severe atrophy of the white matter of the midbrain, pons, and cerebellum than AD [17]. Additionally, DLB is characterized by atrophy of the white matter of the pons [18]. However, our results showed that in patients classified as having $\mathrm{AD}$, the extent of gray matter atrophy and the severity of VOI atrophy in the dorsal brainstem gray matter were significantly negatively correlated. Using VSRAD advance, the area of interest was set in the medial temporal region-which includes the entorhinal cortex, amygdala, and hippocampus-to identify the findings suggestive of AD. The dorsal brainstem gray matter atrophies when the atrophy ratio is high, i.e., at an early stage where atrophy in the MTL selectively progresses, and gray matter atrophy does not. However, when the atrophy ratio is low, i.e., at a stage where selectivity in the MTL decreases and gray matter atrophy progresses, atrophy in the dorsal brainstem gray matter is less likely to occur. Based on this, patients classified as having $\mathrm{AD}$, the dorsal brainstem gray matter atrophies when the gray matter atrophy is mild-which suggests that the dorsal brainstem gray matter could be relatively preserved as gray matter atrophy progresses. In previous studies, brainstem atrophy was observed in patients with $\mathrm{AD}$ [19] and early $\mathrm{AD}$ [20]. The volume of the brainstem is lower in patients with AD than in healthy subjects [20]. Our results are consistent with these reports, suggesting that atrophy of the brainstem is also observed in $\mathrm{AD}$, and that gray matter atrophy is pronounced at a relatively early stage. However, in patients classified as having DLB, the extent of gray matter atrophy and the severity of VOI atrophy in the dorsal brainstem white matter showed a significant correlation. This suggests that in DLB, the dorsal brainstem white matter also atrophies as the gray matter atrophy progresses. It has been suggested that in DLB, the pathway of the Lewy bodies and $\alpha$-synuclein starts from the olfactory mucosa and continues to the cerebral cortex via the hippocampus and amygdala. It then extends to the dorsal nucleus of the vagus in the medulla oblongata via the autonomic nerves, pontine tegmentum, midbrain substantia nigra, and cerebral cortex [21]. Our results may reflect this "break" hypothesis, suggesting that Lewy bodies may develop in the cerebral cortex as the atrophy of the dorsal brainstem progresses-causing further cerebral atrophy. In this study, the extent of gray matter atrophy was $5.7 \%$ and $5.5 \%$ in the patients classified as having AD and DLB respectively. These results indicate that both $\mathrm{AD}$ and $\mathrm{DLB}$ have an almost equal extent of gray matter atrophy. However, patients classified as having $\mathrm{AD}$ and DLB had different results for gray matter and dorsal brainstem atrophy, which may reflect the difference in pathological conditions. Furthermore, it was found that the severity of the dorsal brainstem atrophy varied depending on the state of gray matter atrophy. This 
indicates that there is a need to consider the state of gray matter atrophy when conducting the differential diagnosis between AD and DLB.

There were several limitations in this study that should be considered. First, $\mathrm{AD}$ and DLB were classified based on VSRAD advance values. ASL analysis showed no statistically significant differences in cerebral blood flow patterns between AD and DLB. This may be due to several reasons: 1) ASL resolution is not high; and 2) only one radiologist (have other specialty) evaluated the MRI results. Moreover, VSRAD advance has a sensitivity of $50.6 \%$, specificity of $75.6 \%$, and a correct diagnosis rate of $65.2 \%$ for the differential diagnosis between $A D$ and DLB [11]. Its diagnostic capability is also limited. It is important to note that $\mathrm{AD}$ and DLB can coexist. AD-like changes have also been observed in DLB [22]. Cognitive function declines the fastest in persons with both DLB and AD [23] and is also the poorest in such patients [24]. Therefore, the possibility of distinguishing between AD and DLB may have been limited. Second, based on SWI in MRI, it has recently been reported that in DLB, high intensity areas in the dorsal substantia nigra reflecting melanin disappear [25], and high intensity reflecting melanin in the locus coeruleus decreases [26]. Furthermore, in a study using VS$\mathrm{RAD}$, atrophy of the entorhinal cortex was found to be milder in DLB than in AD [27]. However, the Philips Ingenia 1.5T (Japan, Tokyo) used in this study needed setting changes for melanin imaging. Third, we did not perform psychological tests since quantitative evaluation via VSRAD advance was the focus of this study. However, a correlation between Neurobehavioral Cognitive Status Examination results and $\mathrm{Z}$ score was recently found using the VSRAD [28]. Thus, when studying dementia, it may be necessary to apply psychological testing and utilize neurological examinations. Finally, we had a small sample of participants because this was a single-center retrospective study. Using VSRAD, it was recently found that there is an association between a patient's number of teeth and gray matter atrophy [29]. Thus, there is a need for prospective trials with a greater number of participants using various tests, such as psychological indicators, neurological examinations, and nuclear medicine tests.

\section{Conclusion}

This study showed a negative correlation between the extent of gray matter atrophy and the severity of atrophy in the dorsal brainstem gray matter in patients who were identified to have AD using VSRAD advance. Additionally, a positive correlation between the extent of gray matter atrophy and the severity of atrophy in dorsal brainstem white matter was found in patients with DLB. These results indicate that the severity of dorsal brainstem atrophy depends on the extent of gray matter atrophy.

\section{Conflicts of Interest}

The authors declare no conflicts of interest regarding the publication of this paper. 


\section{References}

[1] Meguro, K., Ishii, H., Yamaguchi, S., Ishizaki, J., Shimada, M., Sato, M., et al. (2002) Prevalence of Dementia and Dementing Diseases in Japan: The Tajiri Project. JAMA Neurology, 59, 1109-1114. https://doi.org/10.1001/archneur.59.7.1109

[2] Yamada, T., Hattori, H., Miura, A., Tanabe, M. and Yamori, Y. (2001) Prevalence of Alzheimer's Disease, Vascular Dementia and Dementia with Lewy Bodies in a Japanese population. Psychiatry and Clinical Neurosciences, 55, 21-25. https://doi.org/10.1046/j.1440-1819.2001.00779.x

[3] Matsui, Y., Tanizaki, Y., Arima, H., Yonemoto, K., Doi, Y., Ninomiya, T., et al. (2009) Incidence and Survival of Dementia in a General Population of Japanese Elderly: The Hisayama Study. Journal of Neurology, Neurosurgery \& Psychiatry, 80, 366-370. https://doi.org/10.1136/jnnp.2008.155481

[4] West, S. and Bhugra, P. (2015) Emerging Drug Targets for A $\beta$ and Tau in Alzheimer's Disease: A Systematic Review. British Journal of Clinical Pharmacology, 80, 221-234. https://doi.org/10.1111/bcp.12621

[5] Kim, W.S., Kågedal, K. and Halliday, G.M. (2014) Alpha-Synuclein Biology in Lewy Body Diseases. Alzheimer's Research \& Therapy, 6, Article No. 73.

https://doi.org/10.1186/s13195-014-0073-2

[6] Stubendorff, K., Hansson, O., Minthon, L. and Londos, E. (2011) Differences in Survival between Patients with Dementia with Lewy Bodies and Patients with Alzheimer's Disease-Measured from a Fixed Cognitive Level. Dementia and Geriatric Cognitive Disorders, 32, 408-416. https://doi.org/10.1159/000335364

[7] Orimo, S., Amino, T., Itoh, Y., Takahashi, A., Kojo, T. and Uchihara, T. (2005) Cardiac Sympathetic Denervation Precedes Neuronal Loss in the Sympathetic Ganglia in Lewy Body Disease. Acta Neuropathological, 109, 583-588.

https://doi.org/10.1007/s00401-005-0995-7

[8] McKeith, I., O’Brien, J., Walker, Z., Tatsch, K., Booij, J., Darcourt, J., et al. (2007) Sensitivity and Specificity of Dopamine Transporter Imaging with ${ }^{123}$ I-FP-CIT SPECT in Dementia with Lewy Bodies: A Phase III, Multicentre Study. Lancet Neurology, 6, 305-313. https://doi.org/10.1016/S1474-4422(07)70057-1

[9] Hirata, Y., Matsuda, H., Nemoto, K., Ohnishi, T., Hirao, K., Yamashita, F., et al. (2005) Voxel-Based Morphometry to Discriminate Early Alzheimer's Disease from Controls. Neuroscience Letters, 382, 269-274. https://doi.org/10.1016/j.neulet.2005.03.038

[10] Matsuda, H., Mizumura, H., Nemoto, K., Yamashita, F., Imabayashi, E., Sato, N., et al. (2012) Automatic Voxel-Based Morphometry of Structural MRI by SPM 8 Plus Diffeomorphic Anatomic Registration through Exponentiated Lie Algebra Improves the Diagnosis of Probable Alzheimer Disease. American Journal of Neuroradiology, 33, 1109-1114. https://doi.org/10.3174/ajnr.A2935

[11] Matuda, M., Yokoyama, K., Sato, N., Ito, K., Nemoto, K., Oba, H., Hanyu, H., et al. (2019) Differentiation between Dementia with Lewy Bodies and Alzheimer's Disease Using Voxel-Based Morphometry of Structural MRI: A Multicenter Study. Neuropsychiatric Disease and Treatment, 15, 2715-2722. https://doi.org/10.2147/NDT.S222966

[12] Kogure, D., Matsuda, H., Ohnishi, T., Asada, T., Uno, M., Kunihiro, T., et al. (2000) Longitudinal Evaluation of Early Alzheimer's Disease Using Brain Perfusion SPECT. Journal of Nuclear Medicine, 41, 1155-1162.

[13] Kaneta, T., Nakatuka, M., Nakamura, K., Seki, T., Yamaguchi, S., Tsuboi, M., et al. (2016) Improved Diagnostic Accuracy of SPECT through Statistical Analysis and 
the Detection of Hot Spots at the Primary Sensorimotor Area for the Diagnosis of Alzheimer' Disease in a Community-Based Study: “The Osaki-Tajiri Project”. Clinical Nuclear Medicine, 41, e1-e6. https://doi.org/10.1097/RLU.0000000000000976

[14] Ishii, K., Yamaji, S., Kitagaki, H., Imamura, T., Hirono, N., Mori, E., et al. (1999) Regional Cerebral Blood Flow Difference between Dementia with Lewy Bodies and AD. Neurology, 53, 413-416. https://doi.org/10.1212/WNL.53.2.413

[15] Sato, T., Hanyu, H., Hirao, K., Shimizu, S., Kanetaka, H. and Iwamoto, T. (2007) Deep Gray Matter Hyperperfusion with Occipital Hypoperfusion in Dementia with Lewy Bodies. European Journal of Neurology, 14, 1299-1301. https://doi.org/10.1111/j.1468-1331.2007.01951.x

[16] Imabayashi, E., Yokoyama, K., Tsukamoto, T., Sone, D., Sumida, K., Kimura, Y., et al. (2016) The Cingulate Island Sign within Early Alzheimer's Disease-Specific Hypoperfusion Volumes of Interest Is Useful for Differentiating Alzheimer's Disease from Dementia with Lewy Bodies. EJNMMI Research, 6, Article No. 67. https://doi.org/10.1186/s13550-016-0224-5

[17] Nakatsuka, T., Imabayashi, E., Matsuda, H., Sakakibara, R., Inaoka, T. and Hitoshi, T. (2013) Discrimination of Dementia with Lewy Bodies from Alzheimer's Disease Using Voxel-Based Morphometry of White Matter by Statistical Parametric Mapping 8 Plus Diffeomorphic Anatomic Registration through Exponentiated Lie Algebra. Neuroradiology, 55, 559-566. https://doi.org/10.1007/s00234-013-1138-9

[18] Kantarci, K., Ferman, T.J., Boeve, B.F., Weigand, S.D., Przybelski, S., Vemuri, P., et al. (2012) Focal Atrophy on MRI and Neuropathologic Classification of Dementia with Lewy Bodies. Neurology, 79, 553-560. https://doi.org/10.1212/WNL.0b013e31826357a5

[19] Ji, X., Wang, H., Zhu, M., He, Y., Zhang, H. and Chen, X. (2021) Brainstem Atrophy in the Early Stage of Alzheimer's Disease: A Voxel-Based Morphometry Study. Brain Imaging and Behavior, 15, 49-59. https://doi.org/10.1007/s11682-019-00231-3

[20] Lee, J.H., Ryan, J., Andreescu, C., Aizenstein, H. and Lim, H.K. (2015) Brainstem Morphological Changes in Alzheimer's Disease. Neuroreport, 26, 411-415. https://doi.org/10.1097/WNR.0000000000000362

[21] Braak, H., Del Tredici, K., Rüb, U., de Vos, R.A., Steur, E.N.J. and Braak, E. (2003) Staging of Brain Pathology Related to Sporadic Parkinson's Disease. Neurobiology of Aging, 24, 197-211. https://doi.org/10.1016/S0197-4580(02)00065-9

[22] Wakisaka, Y., Furuta, A., Tanizaki, Y., Kiyohara, Y., Iida, M. and Iwaki, T. (2003) AgeAssociated Prevalence and Risk Factors of Lewy Body Pathology in a General Population: The Hisayama Study. Acta Neuropathologica, 106, 374-382.

https://doi.org/10.1007/s00401-003-0750-x

[23] Kraybill, M.L., Larson, E.B., Tsuang, D.W., Teri, L., McCormick, W.C., Bowen, J.D., et al. (2005) Cognitive Differences in Dementia Patients with Autopsy-Verified AD, Lewy Body Pathology, or Both. Neurology, 64, 2069-2073. https://doi.org/10.1212/01.WNL.0000165987.89198.65

[24] Blanc, F., Mahmoudi, R., Jonveaux, T., Galmiche, J., Chopard, G., Cretin, B., et al. (2017) Long-Term Cognitive Outcome of Alzheimer's Disease and Dementia with Lewy Bodies: Dual Disease is Worse. Alzheimer's Research \& Therapy, 9, Article No. 47. https://doi.org/10.1186/s13195-017-0272-8

[25] Rizzo, G., De Blasi, R., Capozzo, R., Tortelli, R., Barulli, M.R., Liguori, R., et al. (2019) Loss of Swallow Tail Sign on Susceptibility-Weighted Imaging in Dementia with Lewy bodies. Journal of Alzheimer's Disease, 67, 61-65.

https://doi.org/10.3233/jad-180687 
[26] Taniguchi, D., Hatano, T., Kamagata, K., Okuzumi, A., Oji, Y., Mori, A., et al. (2018) Neuromelanin Imaging and Midbrain Volumetry in Progressive Supranuclear Palsy and Parkinson's Disease. Movement Disorders, 33, 1488-1492.

https://doi.org/10.1002/mds.27365

[27] Hayashi, H., Kawakatsu, S., Suzuki, A., Shibuya, Y., Kobayashi, R., Sato, C., et al. (2012) Application of the VSRAD, a Specific and Sensitive Voxel-Based Morphometry, to Comparison of Entorhinal Cortex Atrophy between Dementia with Lewy Bodies and Alzheimer's Disease. Dementia and Geriatric Cognitive Disorders, 34, 328-331. https://doi.org/10.1159/000345792

[28] Oshikubo, G., Akahane, A., Uno, A., Watanabe, Y., Ikebuchi, E., Tochigi, M., et al. (2020) Utility of VSRAD for Diagnosing Alzheimer's Disease in Patients Screened for Dementia. Journal of International Medical Research, 48, 1-10. https://doi.org/10.1177/0300060520917270

[29] Egashira, R., Umezaki, Y., Mizutani, S., Obata, T., Yamaguchi, M., Tamai, K., et al. (2021) Relationship between Cerebral Atrophy and Number of Present Teeth in Elderly Individuals with Cognitive Decline. Experimental Gerontology, 144, Article ID: 111189. https://doi.org/10.1016/j.exger.2020.111189 